\title{
Estimation of Single-Crystal Elastic Moduli from Polycrystalline X-Ray Diffraction at High Pressure: Application to $\mathrm{FeO}$ and Iron
}

\author{
Anil K. Singh, ${ }^{1}$ Ho-kwang Mao, ${ }^{2}$ Jinfu Shu, ${ }^{2}$ and Russell J. Hemley ${ }^{2}$ \\ ${ }^{1}$ Materials Science Division, National Aerospace Laboratories, Bangalore 5600 17, India \\ ${ }^{2}$ Geophysical Laboratory and Center for High-Pressure Research, Carnegie Institution of Washington, \\ 5251 Broad Branch Road N.W., Washington, D.C. 20005-1305
}

(Received 20 February 1997)

\begin{abstract}
X-ray diffraction data obtained under nonhydrostatic compression of a polycrystalline sample yield an estimate of the single-crystal elasticity tensor of the material when analyzed using appropriate equations. The analysis requires as input the aggregate shear modulus from independent measurements. The high-pressure elastic moduli of face-centered-cubic FeO, body-centered-cubic iron ( $\alpha$-Fe), and the pressure-induced hexagonal close-packed iron $(\epsilon-\mathrm{Fe})$ are obtained. This analysis currently provides the only method of determining single-crystal elasticity tensors in the megabar pressure range and of studying elasticity of very high-pressure phases.

[S0031-9007(98)05436-2]
\end{abstract}

PACS numbers: $62.50 .+\mathrm{p}, 61.10 . \mathrm{Eq}, 62.20 . \mathrm{Dc}, 64.70 . \mathrm{Kb}$

Information on the effect of pressure on the propagation of elastic waves in materials is essential for understanding interatomic interactions, mechanical stability of solids, phase transformation mechanisms, material strength, and seismology. In particular, the elasticity of iron-bearing materials at high pressure has become a subject of great experimental and theoretical interest because of the discovery of new phenomena in these materials at high density [1]. Such phenomena in iron oxides are predicted theoretically to arise from pressure-induced changes in electronic and magnetic interactions [2-4]. The high-pressure behavior of elemental iron is also subject of current theoretical and experimental investigation [5-8]. Determination of the elasticity tensors of the iron polymorphs is important for predicting and ascertaining phase stability and transformations among these phases $[5,7,8]$. Moreover, knowledge of the elasticity of the hcp phase $(\epsilon-\mathrm{Fe})$ is important for understanding the reported seismic anisotropy and rotation of the Earth's inner core [9].

Experimental determination of single-crystal stiffness $c_{i j}$ (or compliance $s_{i j}$ ) tensors, which provide a complete description of the macroscopic elastic properties [10], has been feasible only at low to moderate pressures. Conventional measurements have been carried out using ultrasonic methods, but these are generally limited to low pressures, particularly for single-crystal studies (e.g., $<3 \mathrm{GPa}[11,12])$. Brillouin spectroscopy has been the only technique for elasticity tensor determination up to moderate pressures (e.g., $<25 \mathrm{GPa}[13,14]$ ). However, the technique has been successfully applied only to optically transparent single crystals in hydrostatic media but not to metals and semiconductors at these pressures, and its utility at more extreme conditions has not been demonstrated.

Synchrotron $\mathrm{x}$-ray techniques permit diffraction patterns to be measured from polycrystalline or single-crystal samples to several hundred gigapascals (e.g., [15-18]). Lattice parameter compressibilities provide independent equations for the elasticity tensor elements (e.g., 1 equation for the cubic system and up to 6 for triclinic). Such measurements, however, account for only approximately onethird of the equations required to determine the complete tensor (e.g., 3 elements for cubic and 21 for triclinic [10]). These elastic stiffness coefficients also determine the manner in which materials respond to differential stresses. In the past, differential stresses in specimens under pressure were generally considered undesirable because the principal aim of most studies has been to determine properties such as hydrostatic equations of state. Lattice strain theories [19-21] were therefore developed to correct for systematic effects caused by nonhydrostatic stresses [18,22-24].

In fact, diffraction data obtained under nonhydrostatic stress conditions contain a wealth of additional information on elasticity not contained in hydrostatic compression data. Here we show that diffraction measurements on polycrystalline samples under nonhydrostatic compression can be used to determine the single-crystal elasticity tensor. Together with constraints on the uniaxial stress component derived from the aggregate shear modulus, the technique provides input for the remaining equations required to solve for all of the $s_{i j}$. This allows elasticity tensors of metals and semiconductors to be studied for the first time at very high pressures. We use the technique to study high-pressure elasticity of $\mathrm{FeO}, \alpha$-Fe, and $\epsilon$-Fe.

The nonhydrostatic stress field of a randomly oriented polycrystalline aggregate compressed between two anvils can be defined by principal stresses in the radial $\left(\sigma_{1}\right)$ and axial $\left(\sigma_{3}\right)$ directions [19]. The hydrostatic pressure and uniaxial stress components are given by $\sigma_{P}=\left(2 \sigma_{1}+\right.$ $\left.\sigma_{3}\right) / 3$ and $t=\sigma_{3}-\sigma_{1}$, respectively. General for all crystal systems, the $d$ spacing is a function of $\psi$, the angle between the diffracting plane normal and the load direction [19],

$$
d(h k l)=d_{P}(h k l)\left[1+\left(1-3 \cos ^{2} \psi\right) Q(h k l)\right] .
$$


Here $d_{P}(h k l)$ is the $d$ spacing under hydrostatic pressure $\sigma_{P}$, and $Q(h k l)$ is given by

$$
Q(h k l)=(t / 3)\left[\alpha\left\{2 G_{R}(h k l)\right\}^{-1}+(1-\alpha)\left(2 G_{V}\right)^{-1}\right],
$$

where $t$ is to be taken as positive. $G_{R}^{X}(h k l)$ is the shear modulus under the isostress (Reuss) condition of the polycrystal averaged only over the crystallites that contribute to the intensity at the point of observation. $G_{V}$ is the shear modulus under the isostrain (Voigt) condition. Both $G_{R}^{X}(h k l)$ and $G_{V}$ [25] are functions of $s_{i j}$, and $\alpha$ is a fraction that determines the relative weight of isostress and isostrain conditions. We use Eq. (1) to derive a set of equations to solve for the $s_{i j}$ tensor.

Diffraction patterns are measured with the primary $\mathrm{x}$-ray beam from the synchrotron source nearly perpendicular to the diamond-cell axis (Fig. 1). Extending previous radial diffraction techniques [26], we have developed a high-strength Be gasket (transparent to high-energy $\mathrm{x}$ rays) capable of containing samples at very high pressures [27]. The uniaxial stress component in the polycrystalline sample is deliberately enhanced by not using a soft pressure transmitting medium. The effects of stress gradients are minimized by using samples that are small (typically a disk of $10 \mu \mathrm{m}$ thickness and $25 \mu \mathrm{m}$ diameter) in comparison to the diamond culet (e.g., $400 \mu \mathrm{m}$ diameter). Probing the sample with a microfocus x-ray beam (down to $5 \mu \mathrm{m}$ ) [27], we verify that the radial variation of the lattice strain (stress gradient) is negligible in comparison to the directional variation (uniaxial stress). We use the energy dispersive method with a double collimating system to record the diffraction patterns [28]. With this arrangement, only signal from the sample at the interception of the primary and diffracted beams is received by the detector, while signal from the Be gasket $(5 \mathrm{~mm}$ outer diameter) is suppressed [27]. The diamond cell is rotated about an axis $(R)$ perpendicular to the load axis and bisecting the $2 \theta$ diffraction angle (Fig. 1). Diffraction patterns for $h \mathrm{kl}$ 's in the $20-60 \mathrm{keV}$ range are collected at each increment of $\psi$ to determine $d_{P}(h k l)$ and $Q(h k l)$ in Eq. (1).

For the cubic system [19],

$$
Q(h k l)=m_{0}+3 m_{1} \Gamma(h k l),
$$

where

$$
\Gamma(h k l)=\left(h^{2} k^{2}+k^{2} l^{2}+l^{2} h^{2}\right) /\left(h^{2}+k^{2}+l^{2}\right)^{2},
$$

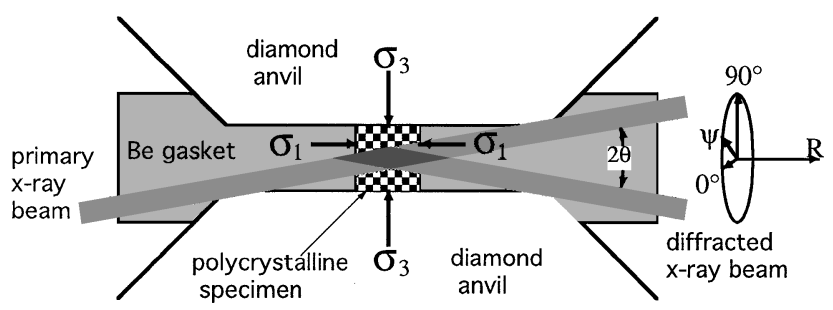

FIG. 1. Geometry for radial x-ray diffraction of the uniaxially compressed sample in a diamond cell.

$$
\begin{gathered}
m_{0}=(\alpha t / 3)\left[\left(s_{11}-s_{12}\right)+\frac{5}{2}\left(\alpha^{-1}-1\right)\right. \\
\left.\times \frac{\left(s_{11}-s_{12}\right) s_{44}}{3\left(s_{11}-s_{12}\right)+s_{44}}\right], \\
m_{1}=-(\alpha t / 3)\left[s_{11}-s_{12}-s_{44} / 2\right] .
\end{gathered}
$$

Equation (6) contains crucial information on $s_{44}$ which is absent in the measurement of hydrostatic compressibility,

$$
\chi_{a}=-(1 / a)(\partial a / \partial P)_{T}=(3 K)^{-1}=s_{11}+2 s_{12} .
$$

Here $K$ is the bulk modulus at $\sigma_{P}$.

The determination of $s_{i j}$ from Eqs. (5)-(7) requires knowledge of $t$. In the absence of an independent gauge, we use the following relation to estimate $t$; this can be derived using the approach given earlier [29]:

$$
t=6 G\langle Q(h k l)\rangle f(x, \alpha),
$$

where

$$
\begin{gathered}
f(x, \alpha)=f_{1} / f_{2}, \\
f_{1}=\frac{2 x+3}{10}+\frac{5 x}{2(3 x+2)}, \\
f_{2}=\alpha[x-3(x-1)\langle\Gamma(h k l)\rangle]+\frac{5 x(1-\alpha)}{3 x+2}, \\
x=2\left(s_{11}-s_{12}\right) / s_{44} .
\end{gathered}
$$

The angle brackets denote the average over all the observed reflections, and $G$ is the aggregate shear modulus at $\sigma_{P}$. For the isotropic case $(x=1), f(x)=1$ and remains close to unity even for reasonably large degrees of anisotropy. However, $f(x)$ can be computed, as $x$ is determined directly from the ratio of $m_{0}$ and $m_{1}$, without requiring knowledge of $t$. For a given set of $m_{0}$ and $m_{1}, x$ is a function of $\alpha$ [Eqs. (5) and (6)]. For different combinations of $\alpha$ and the resulting $x$ (in the range $1 \geq \alpha \geq 0.5$ and $3 \geq x \geq 0.2$ ), the $t$ values calculated from Equation (8) differ by no more than $15 \%$. Equation (8) with $f(x) \approx 1$ is also valid for other crystal systems. The $d(h k l)-\psi$ data fit Eqs. (1) and (3) very well (Fig. 2). The $s_{i j}$ terms for $\mathrm{FeO}$ at $8.3 \mathrm{GPa}$ and $\alpha$-Fe at 4.6 GPa were determined from Eqs. (5)-(7) with $\alpha=1$ and 0.5 . The $t$ values were determined from Eq. (8) using the $G$ estimated by extrapolating (linear in pressure [30]) the ultrasonic data [11,12]. The resulting $c_{i j}$ and $x$ are listed in Table I.

Above $13 \mathrm{GPa}$, iron transforms from bcc to the hcp structure $(\epsilon-\mathrm{Fe})[8,31]$. For the hexagonal system [20],

$$
Q(h k l)=m_{0}+m_{1} B+m_{2} B^{2},
$$

where

$$
B=3 a^{2} l^{2} /\left[4 c^{2}\left(h^{2}+h k+k^{2}\right)+3 a^{2} l^{2}\right],
$$

and $a$ and $c$ are the lattice parameters of the hexagonal 


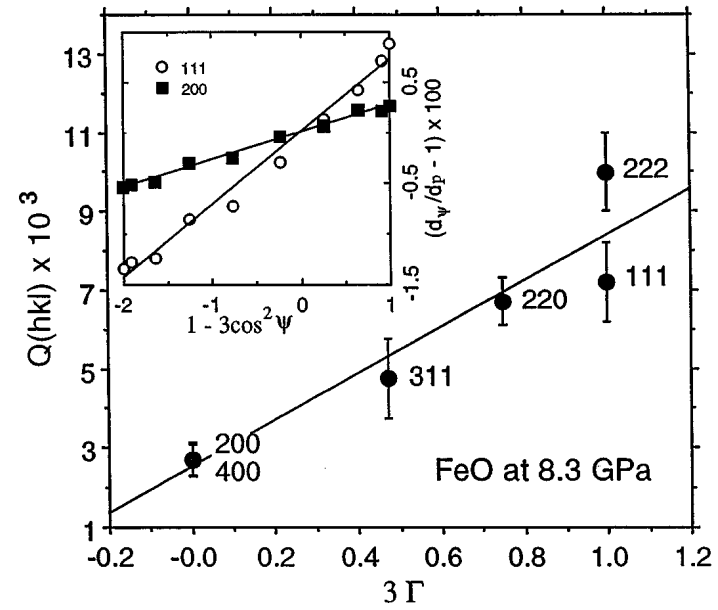

FIG. 2. $Q(h k l)$ versus $3 \Gamma(h k l)$ for $\mathrm{Fe}_{0.98} \mathrm{O}$ at $8.3 \mathrm{GPa}$. A linear relation is observed as predicted by Eq. (3). The slope and intercept are $m_{1}=0.0058(10)$ and $m_{0}=0.0025(7)$, respectively. Inset: $d(h k l)$ versus $\left(1-3 \cos ^{2} \psi\right)$, which gives $d_{P}(111)=2.441(1) \AA, \quad Q(111)=0.0072(10) ; \quad d_{P}(200)=$ 2.114(1) $\mathrm{A}, Q(200)=0.0027(4)$.

unit cell at $\sigma_{P}$. The $m_{i}$ in Eq. (10) are given by

$$
\begin{gathered}
m_{0}=(\alpha t / 6)\left[2 s_{11}-s_{12}-s_{13}+\left(\alpha^{-1}-1\right)\left(2 G_{V}\right)^{-1}\right], \\
m_{1}=(\alpha t / 6)\left(-5 s_{11}+s_{12}+5 s_{13}-s_{33}+3 s_{44}\right), \\
m_{2}=(\alpha t / 6)\left(3 s_{11}-6 s_{13}+3 s_{33}-3 s_{44}\right) .
\end{gathered}
$$

The measurements show that the $Q(h k l)$ data for $\epsilon$-Fe fit Eq. (9) very well, and exhibit a strong $h k l$ dependence: $Q(h k l)$ has minima along the diagonal [102] and maximum along the $a$ [100] and $c$ [002] axes (Fig. 3). Two additional equations are provided by the axial ( $a$ and $c$ ) compressibilities [31],

$$
\begin{gathered}
\chi_{a}=\alpha\left(s_{11}+s_{12}+s_{13}\right)+(1-\alpha)\left(3 K_{V}\right)^{-1}, \\
\chi_{c}=\alpha\left(s_{33}+2 s_{13}\right)+(1-\alpha)\left(3 K_{V}\right)^{-1} .
\end{gathered}
$$

In the absence of shear modulus data on $\epsilon$-Fe, $G$ was obtained by extrapolating ultrasonic data on $\alpha$-Fe. The shear modulus is expected to change across the $\alpha \rightarrow \epsilon$ transition, introducing some uncertainty in $t$. The $s_{i j}$ elements were calculated from Eqs. (11)-(15) for $\alpha=1$ and 0.5 , and the resulting $c_{i j}$ are listed in Table I.

The actual stress state (value of $\alpha$ ) of the sample depends mainly on the magnitude of $t$ in relation to the shear strength of the sample at $\sigma_{P}$. A large value of $t$, the maximum of which equals the sample yield strength, can cause yielding of the crystallites, and this results in the isostress condition $(\alpha=1)$; in contrast, for very small $t, \alpha=0.5$, the lower limit of $\alpha$ [32]. The magnitude of $t$ in turn depends on the extent of plastic flow in the sample [33,34]. The $c_{i j}$ derived with $\alpha=1$ for $\mathrm{FeO}$ and $\alpha$-Fe agree (within experimental error) with the corresponding values obtained from extrapolation of the ultrasonic data [11,12]. With $\alpha=0.5, c_{i j}$ for $\mathrm{FeO}$ show larger deviations from the extrapolated ultrasonic data. In the case of $\alpha$-Fe, agreement for $c_{11}$ and $c_{12}$ improves but marginally worsens for $c_{44}$. The value of $x$ obtained from $m_{0}$ and $m_{1}$ is sensitive to the value of $\alpha$. For a given set of $m_{0}$ and $m_{1}$, the degree of inferred elastic anisotropy is lowest for $\alpha=1$ and increases with decreasing $\alpha$. However, the changes in the individual $c_{i j}$ brought about by changing $\alpha$ from 1 to 0.5 are still within experimental error. Analyses of diffraction data on $\mathrm{Au}$ to 4 GPa show similar trends [35].

The results for $\mathrm{FeO}$ indicate considerable softening of $c_{44}$ at $8.3 \mathrm{GPa}$, a trend also supported by the ultrasonic measurements [11]. The results on $\alpha$-Fe do not indicate softening of $c_{44}$ at $4.6 \mathrm{GPa}$, as found in some bccstructured materials [36]. It has been predicted that at higher pressure the modulus $c^{\prime}=\frac{1}{2}\left(c_{11}-c_{12}\right)$ should soften and vanish above a critical pressure; that is, the reentrant bcc phase proposed at higher pressure should be unstable with respect to a tetragonal distortion [5,7]. Notably, the measured $c^{\prime}$ also increases with pressure

\begin{tabular}{|c|c|c|c|c|c|c|c|c|c|}
\hline & & $8.3 \mathrm{GPa})$ & & & $\mathrm{Fe}(4.6 \mathrm{G}$ & & & e $(52 \mathrm{GPa}$ & \\
\hline & $\alpha=0.5$ & $\alpha=1$ & Ultrasonic $^{\mathrm{a}}$ & $\alpha=0.5$ & $\alpha=1$ & Ultrasonic $^{\mathrm{b}}$ & $\alpha=0.5$ & $\alpha=1$ & Theory $^{\mathrm{c}}$ \\
\hline$c_{11}$ & $413(73)$ & $313(44)$ & $283(5)$ & $260(17)$ & 281(18) & $262(5)$ & $552(65)$ & $639(55)$ & 638 \\
\hline$c_{12}$ & $77(37)$ & $123(22)$ & $144(2)$ & $154(14)$ & $144(12)$ & $155(2)$ & $335(60)$ & $300(55)$ & 190 \\
\hline$c_{13}$ & $\ldots$ & $\ldots$ & $\ldots$ & $\ldots$ & $\ldots$ & $\ldots$ & $301(45)$ & $254(41)$ & 218 \\
\hline$c_{33}$ & $\cdots$ & $\cdots$ & $\cdots$ & $\cdots$ & $\cdots$ & $\cdots$ & $562(80)$ & $648(83)$ & 606 \\
\hline$c_{44}$ & $20(4)$ & $28(7)$ & $36(1)$ & $153(40)$ & $123(30)$ & $128(2)$ & $395(30)$ & $422(23)$ & 178 \\
\hline$c^{\prime}$ & $168(41)$ & $93(25)$ & $78(13)$ & $53(11)$ & $68(11)$ & $53(3)$ & $\ldots$ & $\ldots$ & $\ldots$ \\
\hline$t$ & $1.6(2)$ & $1.4(2)$ & $\cdots$ & $1.3(1)$ & $1.2(1)$ & $\cdots$ & $4.6(2)$ & $4.4(2)$ & $\cdots$ \\
\hline$x$ & $0.12(3)$ & $0.3(1)$ & $0.52(2)$ & $2.9(8)$ & $1.8(6)$ & $2.4(1)$ & $3.6(1.5)$ & $2.5(6)$ & 0.79 \\
\hline
\end{tabular}
from $48 \mathrm{GPa}$ [12] at 1 bar to $68(11) \mathrm{GPa}(\alpha=1)$ [53 GPa $(\alpha=0.5)]$ at $8.6 \mathrm{GPa}$.

TABLE I. $\quad c_{i j}$ of $\mathrm{FeO}, \alpha$-Fe, and $\epsilon$-Fe at high pressure. The standard errors (in parenthesis) were derived from the errors in $m_{i}$; pressure and modulus units in GPa.

${ }^{\mathrm{a}}$ Extrapolation of ultrasonic measurements to $3 \mathrm{GPa}$ in Ref. [11].

${ }^{\mathrm{b}}$ Extrapolation of ultrasonic measurements to $1 \mathrm{GPa}$ in Ref. [12].

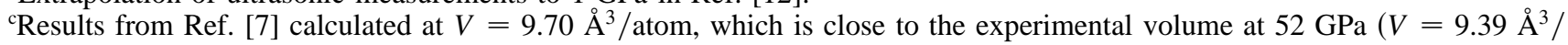
atom) [31]); similar results were obtained in Ref. [6]. 


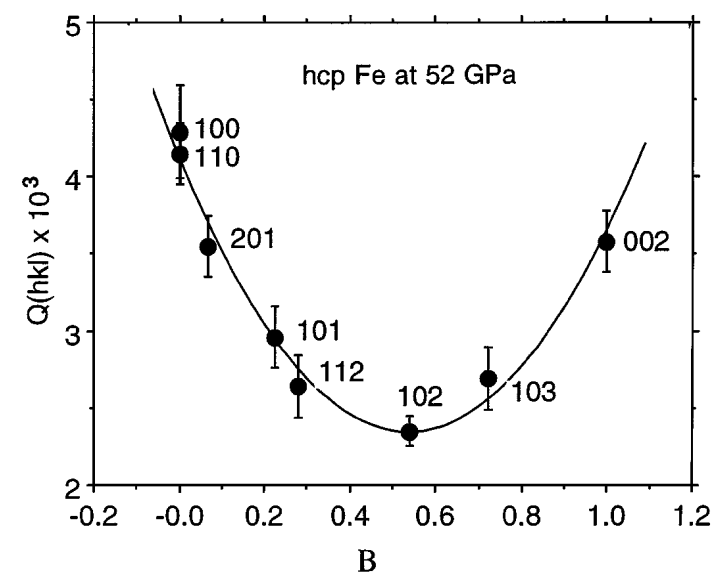

FIG. 3. The $Q(h k l)$ versus $B$ for $\epsilon$-Fe (hcp) at $52 \mathrm{GPa}$. A parabolic relation is observed as predicted by Eq. (9). The bestfit parameters are $m_{0}=4.13(1) \times 10^{-3}, m_{1}=-6.63(5) \times$ $10^{-3}$, and $m_{2}=6.15(7) \times 10^{-3}$.

For $\epsilon$-Fe, the results obtained with $\alpha=1$ agree within experimental error with GGA calculations [7] for $c_{11}, c_{33}$, and $c_{13}$, but disagree markedly for $c_{12}$ and $c_{44}$. The $\alpha=$ 1 analysis gives the lowest estimate of $c_{44} / c_{66}$ (value of 2.5 ), a result that deviates significantly from the condition $c_{44} \approx c_{66}$, which is satisfied by most hcp metals. Our calculations indicate that $c_{44} / c_{66}$ is nearly independent of $t$, and therefore a large value of the ratio cannot be explained by any errors in the estimate of $t$. The relatively high $c_{44} / c_{66}$ ratio requires further examination as it has important implications for interpreting the reported anisotropy of the Earth's inner core.

We are grateful to R.E. Cohen and J. Moriarty for discussions, and to the NSF for support. The NSLS is supported by the DOE (DE-AC02-76-CH00016).

[1] R. J. Hemley and R.E. Cohen, Philos. Trans. R. Soc. London A 354, 1461 (1996).

[2] D. G. Isaak, R.E. Cohen, M. J. Mehl, and D. J. Singh, Phys. Rev. B 47, 7720 (1993).

[3] D. M. Sherman and H. J. F. Jansen, Geophys. Res. Lett. 22, 1001 (1995).

[4] R. E. Cohen, I. I. Mazin, and D. E. Isaak, Science 275, 654 (1997).

[5] L. Stixrude, R. E. Cohen, and D. J. Singh, Phys. Rev. B 50, 6442 (1994).

[6] L. Stixrude and R. E. Cohen, Science 267, 1972 (1995).

[7] P. Soderlind, J. A. Moriarty, and J. M. Wills, Phys. Rev. B 53, 14 (1996).

[8] C. S. Yoo et al., Phys. Rev. Lett. 70, 3931 (1993); S. K. Saxena et al., Science 269, 1703 (1995); C. S. Yoo et al., ibid. 270, 1473 (1995).

[9] X.D. Song and P. G. Richards, Nature (London) 382, 221 (1996).

[10] J.F. Nye, Physical Properties of Crystals (Clarendon Press, Oxford, 1985).

[11] I. Jackson, S. K. Khanna, A. Revcolevschi, and J. Berthon,
J. Geophys. Res. 95, 21671 (1990).

[12] M. W. Guinan and D. N. Beshers, J. Phys. Chem. Solids 29, 541 (1968).

[13] C. S. Zha, T. S. Duffy, H. K. Mao, and R. J. Hemley, Phys. Rev. B 48, 9246 (1993).

[14] H. Shimizu, M. Ohnishi, S. Sasaki, and Y. Ishibashi, Phys. Rev. Lett. 74, 2820 (1995).

[15] H. K. Mao et al., Phys. Rev. Lett. 64, 1749 (1990).

[16] Y. K. Vohra and J. Akella, Phys. Rev. Lett. 67, 3563 (1991).

[17] A. L. Ruoff, H. Xia, and Q. Xia, Rev. Sci. Instrum. 63, 4342 (1992).

[18] T. S. Duffy, R. J. Hemley, and H. K. Mao, Phys. Rev. Lett. 74, 1371 (1995).

[19] A. K. Singh, J. Appl. Phys. 73, 4278 (1993).

[20] A. K. Singh and C. Balasingh, J. Appl. Phys. 75, 4956 (1994).

[21] T. Uchida, N. Funamori, and T. Yagi, J. Appl. Phys. 80, 739 (1996).

[22] N. Funamori, T. Yagi, and T. Uchida, J. Appl. Phys. 75, 4327 (1994).

[23] Y. Meng, D. J. Weidner, and Y. Fei, Geophys. Res. Lett. 20, 1147 (1993).

[24] R. Jeanloz, B. K. Godwal, and C. Meade, Nature (London) 349, 687 (1991).

[25] R. F. S. Hearman, Adv. Phys. 5, 323 (1956).

[26] G. L. Kinsland and W. A. Bassett, Rev. Sci. Instrum. 47, 130 (1976); J. Appl. Phys. 48, 978 (1977). In these experiments, the $\mathrm{x}$-ray beam measured the entire radial stress gradient in the sample, which can introduce an error comparable or larger than the deviatoric stress.

[27] H. K. Mao and R. J. Hemley, High Press. Res. 14, 257 (1996); R. J. Hemley et al., Science 276, 1242 (1997).

[28] The experiments were carried out at the superconductor wiggler beam line X17C of the National Synchrotron Light Source; see Ref. [27].

[29] A. K. Singh, Philos. Mag. Lett. 67, 379 (1993).

[30] For $\mathrm{FeO}, G_{0}=46.4 \mathrm{GPa}$ [11], and extrapolation with a second degree polynomial [11] to $8.3 \mathrm{GPa}$ gives $G=$ $45.6 \mathrm{GPa}$. For $\alpha$-Fe $\left(G_{0}=82 \mathrm{GPa}\right.$ [12]), linear extrapolation in pressure gives 90 and $175 \mathrm{GPa}$ at 4.6 and $52 \mathrm{GPa}$, respectively. At high pressures, where linear extrapolation is inadequate, equations based on finite strain theory may be used [e.g., G.F. Davies and A.M. Dziewonski, Phys. Earth Planet. Inter. 10, 336 (1975)]. The value of $t$ for $\epsilon$-Fe at $52 \mathrm{GPa}$ is calculated using $G$ from linear extrapolation only because of the uncertainty introduced by a possible discontinuity in $G$ at the $\alpha \rightarrow \epsilon$ transition (see text).

[31] A. P. Jephcoat, H. K. Mao, and P. M. Bell, J. Geophys. Res. 91, 4677 (1986).

[32] R. Hill, Proc. Phys. Soc. London Sect. A 65, 349 (1952).

[33] C. Meade and R. Jeanloz, J. Geophys. Res. 93, 3261 (1988).

[34] A study of $\mathrm{NaCl}$ [22] concluded that $\alpha=1$ at low pressures, and progressively shifts to $\alpha=0.5$ as the pressure is increased. For reasons given in the text, this trend cannot be generalized.

[35] H. K. Mao et al. (to be published).

[36] M. Born and K. Huang, Dynamical Theory of Crystal Lattices (Oxford, London, 1954). 